\title{
Probing Ultralow Energy Excitations at Ultrahigh Spatial Resolution with Monochromated Electron Energy Loss Spectroscopy
}

\author{
Jordan Hachtel
}

Center for Nanophase Materials Sciences, Oak Ridge National Laboratory, United States

Electron energy loss spectroscopy (EELS) in a scanning transmission electron microscope (STEM) is a technique can access a wealth of information with a nanoscale probe. However, the ultimate energy resolution in an EELS experiment is limited by variation in energies of the electrons emitted from the electron source. The best electron sources available in modern S/TEMs have energy spreads on the order of $\sim 300 \mathrm{meV}$, which is totally prohibitive for ultralow energy excitations, such as phonons or molecular vibrations, which can have line widths of under a single meV. Additionally, there is a dominant elastic scattering peak centered around an energy loss of zero, and the tail of this peak in conventional STEM experiments creates a massive background that extends into the visible, further obscuring infrared analyses.

In order to access infrared, we utilize a technique called electron monochromation, where the beam is dispersed by energy and only a narrow band is allowed to pass through. Modern monochromated STEMs (see Fig. 1a) can reach an energy resolution as low as $3 \mathrm{meV}$ [1], which is opens up the door to a wide range of experiments in biology and materials science that were not feasible at normal energy resolution.

Comparisons between the normal ZLP of a conventional cold field emission gun (cFEG) and the ZLP at a high degree of monochromation are shown in Fig. 1b and in log-scale in Fig. 1c. Here, we can see that without monochromation the range of energies coming out of the electron gun is far too broad to detect phonons in the narrow phonon band of a material such as hexagonal boron nitride $(\mathrm{hBN})$, however with monochromation the ZLP width and tail are sufficiently reduced to the point where the low-intensity narrow-linewidth excitations are easily observable. The combination of improved energy resolution and reduced background enables the ultralow excitations in the infrared to be accessed at the native energy resolution of the electron probe [2].

However, accessing this regime is only half the battle. Many new challenges arise when attempting to use EELS to access the nanoscale response of these excitations. In this tutorial, I will cover basic operation of the monochromated STEM, as well as several different classes of experiments that are possible with the new combination of energy and spatial resolution available therein. Specifically, I will cover:

- Aloof Spectroscopy: Here, the evanescent field of the electron beam is used to gently probe beam sensitive samples without directly irradiating it with the high-energy electrons in the beam. The energy resolution is sufficient to identify vibrational modes and isotopic labels in beam-sensitive molecules [3-5]. Moreover, while delocalized, the beam still possesses nanometer scale resolution, which has significant implications in the biological sciences as it would allow the direct analysis of beamsensitive samples (such as frozen hydrated whole-cell samples) possessing nanoscale complexity.

- Off-Axis Spectroscopy: The aloof effect can also negatively impact spatial resolution, since at any beam position the measured EELS signal is still strongly modified by the aloof interaction with the surrounding area. However, one can regain the native atomic-resolution of STEM through using the off-axis geometry, where we only collect EELS from high-angles away from the optic axis. The offaxis geometry allows for atomic-resolution vibrational EELS [6], even going down to the single atom level [7], allowing effective probing of interfaces and nanostructures [8].

- Momentum-resolved Spectroscopy: The ability to control the collection angles used for off-axis spectroscopy can be combined with manipulation of the convergence angles of the probe to achieve simultaneously high spatial-, spectral- and momentum resolution. By choosing small convergence 
angles the electron diffraction pattern can be used to define the Brillouin zone allowing careful collection of the EELS signal at specific values in momentum space while retaining nanoscale resolution $[9,10]$. This enables the direct efficient collection of phonon dispersions with nanometer scale resolution.

\section{Acknowledgements}

Research conducted at the Center for Nanophase Materials Sciences, which is a DOE Office of Science User Facility. Work was performed, in part, using instrumentation within ORNL's Materials Characterization Core provided by UT-Battelle, LLC, under Contract No. DE-AC05- 00OR22725 (J.C.I.) with the DOE, and sponsored by the Laboratory Directed Research and Development Program of Oak Ridge National Laboratory, managed by UT-Battelle, LLC, for the U.S. Department of Energy.
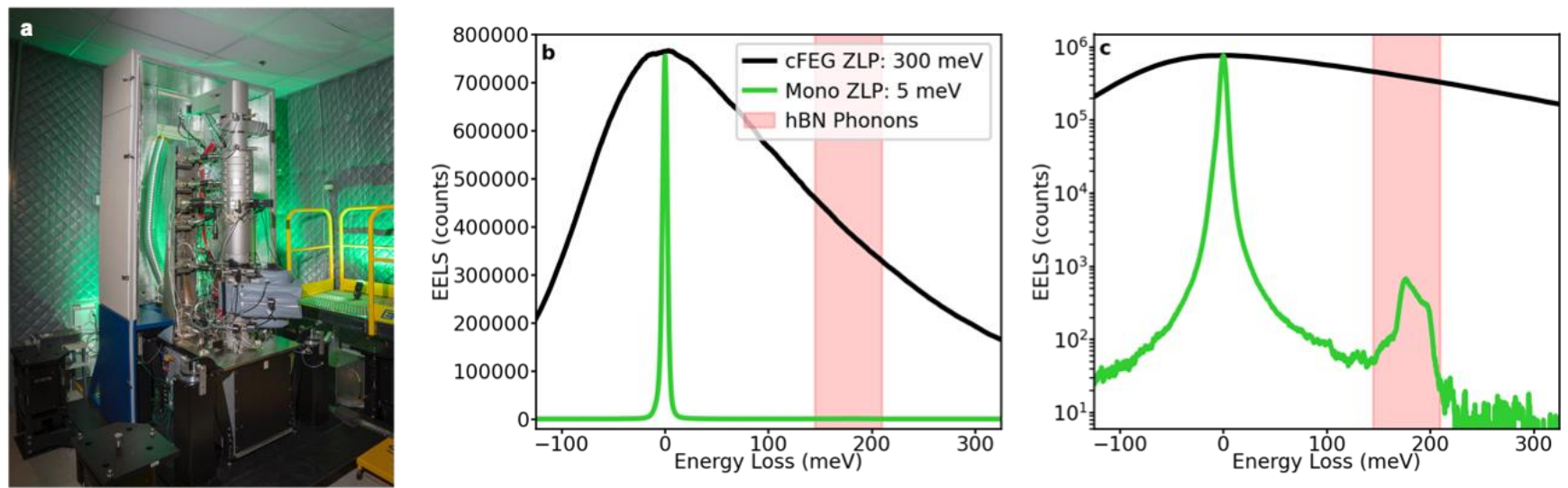

Figure 1. Figure 1. Monochromated EELS in the STEM. (a) Image of a Nion High-Energy-Resolution Monochromated EELS STEM (HERMES) capable of ultrahigh energy resolution EELS. (b,c) Comparison of a ZLP without monochromation (black) to a ZLP with monochromation (green) showing the significant reduction in absolute width of the energy spread and the tail extending through the infrared in linear (b) and $\log$ (c) scale. Both samples are acquired on hexagonal boron nitride (hBN), whose phonons are all present in the Reststrahlen band from 150-200 meV. The phonons are clearly visible with monochromation but impossible to observe without.

References

[1] OL Krivanek et al., Ultramicroscopy 203 (2019), p. 60

[2] JA Hachtel, AR Lupini and JC Idrobo, Sci. Rep. 8 (2018), p. 1

[3] DM Haiber and PA Crozier, ACS Nano 12 (2018) p. 5463

[4] JR Jokisaari et al., Adv. Mater. 30 (2018) p. 1801286

[5] JA Hachtel et al., Science 363 (2019) p. 525

[6] FS Hage et al., Phys. Rev. Lett. 122 (2019) p. 016103

[7] FS Hage et al., Science 367 (2020) p. 1124

[8] X Yan et al., Nature 589 (2021) p. 65

[9] FS Hage et al., Sci. Adv. 4 (2018) eaar7495

[10] R Senga et al., Nature 573 (2019) p. 247 